\title{
IMPACTO DE LA ADOPCIÓN DE UNA MASCOTA EN LAS PERCEPCIONES DE BIENESTAR FÍSICO Y EMOCIONAL ${ }^{1}$
}

\section{The Impact of Adopting a Pet in the Perception of Physical and Emotional Wellbeing}

\author{
Mariana Londoño Taborda ${ }^{2}$, Mariantonia Lemos ${ }^{3} \mathrm{Y}$ \\ JohnNy JaVier Orejuela ${ }^{4}$
}

Recibido: 2018-07-03 Aceptado: 2018-12-12

\begin{abstract}
Resumen. Este estudio indaga por las percepciones de un grupo de personas que van a adoptar una mascota — gatos o perrossobre éstas, así como por las percepciones de bienestar emocional y físico influenciadas por la presencia de la mascota en sus vidas. Es un estudio cualitativo fenomenológico, con entrevista semiestructurada, aplicado sobre nueve personas en el momento de realizar la adopción de su mascota, y tres meses después, cuando ya estaban conviviendo con éstas. Se encontró que aquellos que se referían a su mascota como un animal tendían a adoptar para que éste sirviera de compañía o ayudara en los procesos de responsabilización de un miembro de la familia, mientras que aquellos que veían a la mascota como un miembro más de la familia lo buscaban por compañía $\mathrm{y}$ amor, y asimismo manifestaron mayor im-
\end{abstract}

pacto en sus percepciones sobre el bienestar físico y emocional. Cabe anotar que la percepción sobre las mascotas tendió a cambiar en el tiempo, pasando de verlas sólo como un animal hasta darles un lugar en la familia. Finalmente, se encontró que los participantes desarrollaron en general una relación empática con sus mascotas, preocupándose por sus necesidades y modificando sus conductas para darle bienestar al animal de compañía, aspecto que también influyó en su propio bienestar.

Palabras clave: animal, salud, rol, relación.

Abstract: The aim of this study was to explore the perceptions of a group of individuals that are going to adopt a pet (cat or dog), as well as the perceptions of physical and emotional wellbeing that they consider have been
Para citar este artículo en APA: Londońo, M., Lemos, M. y Orejuela, J. (2018). Impacto de la adopción de una mascota en las percepciones de bienestar físico y emocional. Revista de Psicología Universidad de Antioquia, 10(2), 53-74. DOI: 10.17533/udea.rp.v10n2a03
1. Artículo derivado de la investigación "Impacto en las percepciones de salud y bienestar que se generan en un grupo de personas después de adoptar una mascota”, realizada durante los ańos 2017-2018.

2. Psicóloga, Universidad EAFIT, Medellín, Colombia. Correo: mlondo55@eafit.edu.co; https://orcid.org/0000-0002-34050044 .

3. Doctora en Psicología. Profesora Pregrado de Psicología, Universidad EAFIT. Correo: mlemosh@eafit.edu.co; http:// orcid.org/0000-0002-9131-4704.

4. Doctor en Psicología social del trabajo. Profesor Pregrado de Psicología, Universidad EAFIT. Correo: jorejue2@eafit.edu. co. https://orcid.org/0000-0001-9181-463X. 
influenced by the presence a pet in their lives. A qualitative phenomenological study was carried out between 2017 and 2018 with a sample of nine people recruited at the moment of adoption for a semi structured interview and a follow-up three months later. Results show that individuals that referred to their pet as an animal generally adopted in order to have company or to help in the processes of responsibility of a member of the family, while those that saw the pet as a member of the family adopted for love and companionship, but also reported a greater impact in their per- ceptions of physical and emotional wellbeing. It is worth noted that the perception of the pet tended to change during time, since "just an animal" to someone that has a role in the family. Finally, it was found that participants generally developed an empathic relationship with their pets, caring for their needs and modifying behaviors in order to provide them wellbeing, which in turn influenced their own wellbeing.

Keywords: animal, health, role, relationship.

\section{Introducción}

Actualmente en Colombia en seis de cada diez hogares hay una mascota o animal de compañía, por lo general perros y gatos (El Espectador, 2015). Entre las razones más comunes que las personas brindan para tenerlos está el hecho de que los animales ofrecen compañía incondicional, dan sensación de cuidado y protección a sus cuidadores y distraen a las personas de sus preocupaciones cotidianas (Revista 4 Patas, 2017). Sin embargo los estudios van más allá y han indagado por los efectos de los animales en el contexto terapéutico de la terapia asistida por animales (Ávila et al., 2013), equinoterapia (Ramos, Díaz, Báez y Tarajano, 2006), zooterapia (Fonseca, 2010) y delfinoterapia (Pineda, 2008), y se han centrado principalmente en los beneficios de la tenencia de animales, ya sea en la población en general (Hugues et al., 2014a), en pacientes con enfermedades cardiovasculares (Hugues et al., 2014b) o en adultos mayores con diabetes mellitus de tipo 2 (Hugues et al., 2016).

Igualmente, se ha encontrado que tener perros aumenta los niveles de actividad física por parte de sus dueńos (Díaz y Olarte, 2016; Kushner, Jackson, Jewell y Rudloff, 2006; Lewis, Krägeloh y Shepherd, 2009; Rodríguez, 2013). $\mathrm{Al}$ respecto, Lutz (2013) afirma que este incremento en la actividad física lleva a que los dueños de las mascotas sean menos propensos a tener enfermedades como la obesidad, artritis o un diagnóstico de insuficiencia cardiaca congestiva en comparación con quienes no tienen una mascota. Sin embargo, este estudio también evidenció que las personas que tienen mascotas presentan una mayor tendencia a sufrir de alergias y asma. En relación con las personas 
con enfermedades, se ha hallado que en personas con cardiopatías las mascotas cumplían un papel motivador sobre el aumento de la salud física en actividades como caminatas y las personas consideraban que ellos les ayudaban a controlar y manejar su enfermedad (Hugues et al., 2014a, Rodríguez y Muñoz, 2010).

Adicionalmente, en adultos de la tercera edad se ha encontrado que la tenencia de mascotas se relaciona con mayor actividad, comida más saludable y más cuidado de su salud, ya que en varios casos los animales que compartían con ellos se ponían inquietos o tenían actitudes diferentes ante alteraciones del balance corporal, como cuando tales adultos sufrían episodios de hipoglicemia (López, 2015; Hugues et al., 2016; Pacheco, 2012). Otros estudios han evidenciado la influencia positiva que tienen los perros en temas como la disminución del estrés y del cortisol que presentan las personas, lo cual propicia el aumento de la salud mental de los mismos (Polheber y Matchock, 2013; Gonzáles y Landero, 2011), pues al someter a los voluntarios a situaciones generadoras de estrés, el grupo que estaba en compañía de un perro mostraba menores niveles de cortisol en su sistema en comparación con los voluntarios que estaban acompañados de un amigo o estaban solos.

Asimismo, autores como Núñez, León, Morales y Roa (2004) evidenciaron que la tenencia de animales de compañía en adultos mayores se relaciona directamente con el optimismo que dichas personas tienen sobre la vida, lo cual explicaría el mejor estado de salud que éstos presentan respecto a un grupo de personas sin mascotas. Por último, debe considerarse que estar expuestos a interacciones con mascotas desde temprana edad, específicamente perros, propicia el adecuado desarrollo de la motricidad gruesa de los niños en comparación con quienes no están en contacto con dicho animal. Cabra, Hincapié, Jiménez y Tobón (2011) lograron establecer que los aspectos que se ven beneficiados por dicha interacción son la lateralidad, la noción de cuerpo y las estructuras espaciotemporales.

Estos datos contrastan con el hecho de que en la actualidad, debido al estilo de vida que exige la sociedad — hiperproductivismo, consumismo y fuerte individualización de las relaciones sociales-, el cuidado de sí mismo ha pasado a segundo plano ya que aspectos como lo laboral han pasado a ser 
altamente prioritarios, por lo cual es común encontrar a personas con diferentes enfermedades causadas por el estrés cuando éste sobrepasa los niveles óptimos, generando consecuencias negativas en el ámbito laboral como, por ejemplo, alteraciones en la productividad, en la motivación, la satisfacción, el bienestar, el compromiso y la salud mental, así como trastornos gastrointestinales, cardiovasculares, ansiedad, angustia, depresión, entre otros (Durán, 2010; Ivancevich y Matteson, 1992; Orejuela, 2018).

Dado lo anterior, y considerando que la Organización Mundial de la Salud - OMs - define la salud como "un estado de completo bienestar físico, mental y social, y no solamente la ausencia de afecciones o enfermedades» (oms, 2015), se hace relevante investigar diferentes alternativas que puedan contribuir a mejorar el estado de salud de las personas de forma integral, yendo más allá de las enfermedades y centrándose en el bienestar social, biológico y emocional con el que las personas deberían contar. Estas perspectivas están en pro de la calidad de vida de las personas en términos de condiciones de vida y satisfacción con ella (Urzúa, 2012).

Cabe anotar que la evidencia encontrada hasta el momento sobre las mascotas se orienta en gran medida a personas con diversas enfermedades o que se encuentran en la tercera edad. Sin embargo, pocos estudios se orientan al impacto que generan las mascotas en el bienestar y la calidad de vida de la población en general. De esta manera, este estudio pretende aportar información sobre los posibles impactos que tienen los animales de compañía, mejor conocidos como mascotas, en las percepciones de bienestar emocional y personal de sus dueños. De este modo, los resultados que pueda arrojar la investigación presente aportarían al campo del conocimiento psicológico en cuanto a la consideración de los beneficios de implementar animales, no sólo en el proceso terapéutico de una persona, sino también como una estrategia de mejoramiento de su calidad de vida y percepción de bienestar subjetivo a partir del uso creativo del tiempo libre, con el fin de reforzar, optimizar y complementar las técnicas, herramientas y formas de abordaje utilizadas en los ámbitos clínicos y no clínicos - cotidianos-.

Teniendo en cuenta lo anterior, se formularon como objetivos para este estudio: 
1) indagar por las percepciones que tienen sobre las mascotas - gatos y perros - un grupo de personas que contactan alguna fundación del Área Metropolitana del Valle de Aburrá entre el 2017 y 2018 para llevar a cabo un proceso de adopción;

2) explorar de qué manera la adopción de la mascota afecta las percepciones de bienestar emocional y físico de estas personas.

\section{Método}

\section{Diseño}

Para dar respuesta a los objetivos de este estudio, se diseñó un estudio cualitativo fenomenológico en el que se contactó a los posibles participantes, antes de adoptar una mascota en alguna fundación del Área Metropolitana, para invitarlos al estudio. Aquellos que accedieron participar fueron citados para una entrevista semiestructurada que buscaba explorar las razones que llevaban a la decisión de adoptar; asimismo se les pidió autorización para volverlos a contactar tres meses después para una nueva entrevista enfocada en explorar el impacto de las mascotas en sus percepciones de su bienestar emocional y físico.

\section{Participantes}

Los participantes fueron contactados en diferentes fundaciones vinculadas a la adopción de mascotas en el Área Metropolitana. Los participantes debían tener entre 18 y 65 años y no habían de contar con ningún tipo de mascota en el momento de la adopción, es decir, la mascota que adoptaron debía ser la primera que tenían en la vida o en un lapso de mínimo un año. Se excluyeron aquellos sujetos que tuvieran algún tipo de discapacidad cognitiva, neurológica, motora o psiquiátrica que obstaculizara el desarrollo de la entrevista semiestructurada. 
Se evaluaron nueve personas, de las cuales cuatro fueron hombres y cinco mujeres. Una de las participantes vivía sola, mientras que los demás compartían su casa con miembros de su familia nuclear. Las características de los participantes se describen en la tabla 1 .

Tabla 1. Características demográficas de los sujetos evaluados en el estudio

\begin{tabular}{|llllll|}
\hline Sujeto & Sexo & Edad & Estado civil & Con quién vive & Mascota adoptada \\
\hline 1 & Hombre & 54 & Casado & Esposa e hijos & Perro \\
2 & Mujer & 21 & Soltera & Mamá & Gato \\
3 & Mujer & 36 & Casada & Esposo e hijos & Perro \\
4 & Hombre & 49 & Casado & Esposa e hijos & Perro \\
5 & Mujer & 24 & Soltera & Sola & Dos gatos \\
6 & Mujer & 53 & Casada & Esposo e hija & Gato \\
7 & Hombre & 46 & Casado & Esposa e hijos & Perro \\
8 & Mujer & 20 & Soltera & Padres & Perro \\
9 & Mujer & 43 & Casada & Esposo e hijos & Perro \\
\hline
\end{tabular}

\section{Procedimiento}

El proyecto se presentó a diferentes fundaciones para el cuidado y adopción de animales del Área Metropolitana y de esa manera solicitar el acceso a información de los posibles participantes. En aquellas donde el proyecto fue aceptado, cuando los individuos iniciaban el contacto para llevar a cabo la adopción, las personas de las fundaciones les contaban del estudio y los invitaban a participar. Quienes aceptaron la invitación firmaron el consentimiento informado y posteriormente respondieron la entrevista semiestructurada. A todos los participantes se les informó que iban a ser contactados a los tres meses para un seguimiento. La entrevista inicial tomó un tiempo aproximado de 20 minutos y la segunda de 15 minutos.

\section{Análisis de la información}

Las entrevistas fueron transcritas y codificadas inicialmente de acuerdo con las categorías iniciales — razones para adoptar, e impactos esperados y obte- 
nidos en los ámbitos físico y emocional—. El análisis implicó la observación de similitudes y diferencias en las respuestas para encontrar tendencias y dar cuenta de los códigos analíticos. Los análisis cualitativos se desarrollaron en el programa AtLAs.ti versión 7.

\section{Resultados}

\section{Razones para la adopción}

$\mathrm{Al}$ analizar las entrevistas realizadas a los adoptantes en el momento previo a la adopción de la mascota, se encontró que los participantes le otorgaron dos diferentes roles al animal de compañía y que, a su vez, éste influyó directamente en la justificación de la adopción —figura 1—. Para las personas que sólo consideraron a la mascota como un animal, bajo una perspectiva más instrumental, la adoptaron porque pensaron que el animal de compañía ayudaría al logro de algún propósito, por ejemplo: la adquisición de responsabilidades en algunos integrantes de la familia, principalmente los niños, dado que ellos serían los encargados de velar por las demandas de las mascotas y así llegar a ser los responsables de éstas. Al respecto, uno de los participantes comentó que decidieron adoptar:

[...] porque los niños estaban insistiendo en que querían un perrito, adoptar alguna mascotica, como teníamos pescaditos y no les gustaban — todavía los tenemos, inclusive, pero no, [querían] un animalito que pueda compartir más con nosotros. Pensamos en un gato o en un perro y yo les dije que una mascota que por lo menos sea pequeña, entonces más que todo fue por los niños, por darle gusto a ellos... aspiramos [a] que los nińos cojan responsabilidades frente a muchas cosas de ellos, entonces espero que tengan obligaciones [con] la mascotica y con otros quehaceres de la casa, que colaboren con el aseo, que sean más disciplinados con el estudio, en ese sentido cedimos con la mascota (S1, comunicación personal, ago 10, 2017).

Por otra parte, se identificó que otras personas asignaban a la mascota un rol menos instrumental y más trascendental, con tendencia a humanizarlo —atribuyéndole características o rasgos humanos - o asumirlo en la posición de un álter ego como si de otro ser humano se tratara; por ejemplo, un 
participante indicó que «el perrito aquí se va a tratar como otra persona de la casa, porque yo pienso que ellos son como otra persona, es un animalito muy entendido y todo eso y hay que tratarlos como una persona» (S7, comunicación personal, nov 27,2017). A su vez, se encontró que quienes tuvieron la tendencia a humanizar a los animales de compañía adoptaron por dos razones: compañía y gusto. Con respecto a la primera razón, una participante afirmó: «[...] que rico, como una compañía para mi papá porque mi papá mantenía mucho acá, pues mantiene mucho acá en la casa, es encerrado, no sale, entonces principalmente fue como por eso» ( $\mathrm{S} 8$, comunicación personal, nov 28 , 2017), y otra dijo: «[...] también creo que será una gran compañía porque mi casa es sola y creemos que al llegar y encontrar a la mascota será como una compañía» (S2, comunicación personal, sept 12, 2017). La segunda razón se evidenció en expresiones como la dada por una de las participantes, quien comentó que: «[...] no tenemos mascotas en este momento y a mi mamá le gustan mucho los gatos y por esto queremos adoptar uno en vez de un perro» (S2, comunicación personal, sept 12, 2017).

Cabe anotar que tres meses después de la adopción se identificó que los dos roles mencionados anteriormente se mantuvieron. En cuanto al primer grupo de personas, se evidenció el caso de una participante la cual antes de la adopción había comentado: «[...] pues la verdad yo de las mascotas, yo no he sido como muy amiga, las quiero, pero de lejitos... me parecen lindas y todo, pero de lejitos, pero vamos a ver cómo me va con la propia» (S6, comunicación personal, nov 28, 2017); y que tres meses después de la adopción expresó:

[...] las mascotas son muy lindas, yo siempre he dicho que me parecen muy lindas pero fuera, pues yo he sido como enemiga de tener mascotas en mi casa, son muy lindos y no tengo problemas contra ellos, pero ahora que ya tengo uno me he ido como encariñando, no he soltado la rienda total pero sí me he ido como adaptando, lo que pasa es que me genera como cierta... no sé si es miedo o temor, no sé, no sé cómo descifrarlo (S6, comunicación personal, feb 15, 2018).

En lo concerniente a aquellos que tendieron a humanizar a su mascota, se encontró que tres meses después éstas hacían parte de la familia y la trataban como un miembro más. Verbigracia, una participante afirmó que los animales adoptados: «[...] son muy lindos, nosotros los queremos mucho... como dijo 
mi esposo, es otro integrante más acá en la casa» (S3, comunicación personal, nov 2, 2017); en tanto que otro participante indicó: «[...] siento que son como otra persona, están con uno, lo acompañan, juegan con uno» (S7, comunicación personal, feb 6, 2018).

Figura 1. Rol asignado y motivos de la adopción

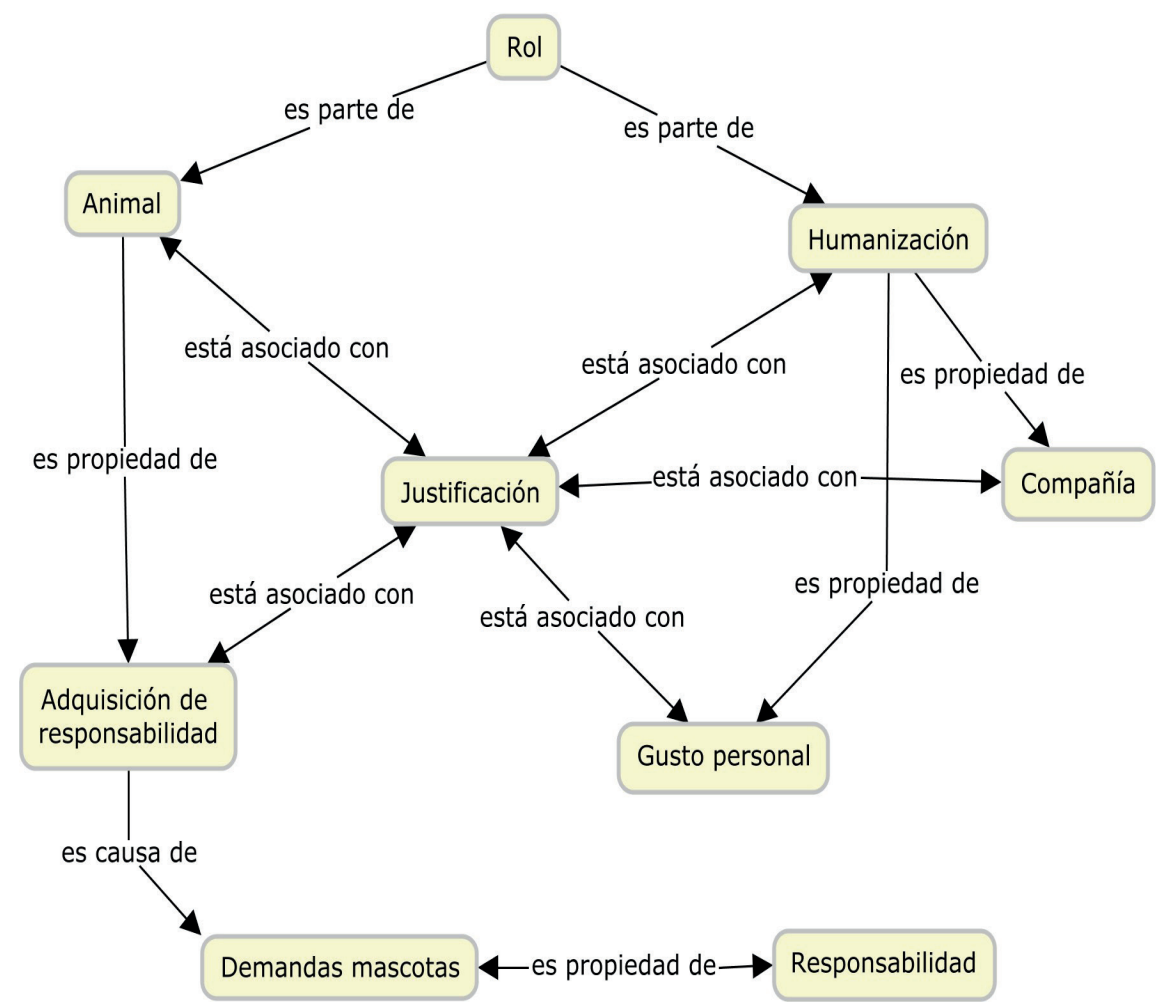




\section{Impacto físico y emocional de las mascotas en los adoptantes}

Tres meses después de la adopción se identificó que el humanizar a la mascota fue fundamental sobre el impacto emocional percibido por los adoptantes. Se evidenció que los participantes que tendieron a humanizar a sus mascotas desarrollaron una relación empática con ellos, la cual se definió como atribución de sentimientos, capacidades o actitudes propias de los humanos a sus animales de compañía. Esto fue patente en expresiones de algunos participantes tales como la siguiente:

[...] desde que nos conocimos hubo empatía, yo lo conocí hace tres meses y hubo química entre los dos, de hecho, es un perro que no es un bebé, tiene dos años y desde que lo traje hubo tanto empatía que la relación es como si nos conociéramos desde hace mucho tiempo (S4, comunicación personal, ene 5, 2018).

Otra participante expresó que:

[...] se siente como si fuera un niño más de la casa, entonces es el que da ternura, es muy tierno, la perrita es toda tierna, entonces uno también se vuelve como más tierno, se vuelve como un niño también, la perrita le mueve a uno la cola, entonces uno empieza a hablarle pues [de manera] mimada a la perrita ( 99 , comunicación personal, ene 5, 2018).

Por último, se evidenció el caso de una participante que consideró que su animal de compañía le daba afecto, porque "puede brindar en cierto modo el mismo cariño que una persona» (S8, comunicación personal, feb 5, 2018).

Sobre el impacto en el ámbito emocional se encontró que una parte está mediado por la compañía que las mascotas ofrecen, ya que algunos participantes expresaron sentimientos de bienestar y acompañamiento derivados de su presencia. V. gr., un participante indicó que «los niños se sienten muy acompañados y por ejemplo llegan a la casa y son alegres de llegar aquí porque saben que la perrita los está esperando» (S9, comunicación personal, ene 5, 2018); otro participante sostuvo que la mascota: «[...] me trae mucha tranquilidad, mayor compañía, me da mucha confianza... las mascotas son la mejor compañía para los seres humanos» (S4, comunicación personal, ene 5, 2018). De igual modo, algunos participantes comentaron que se integraron más y la 
familia se unió en torno a la mascota, es decir, la presencia de la mascota y su cuidado obligó a la interacción e integración social entre los integrantes de la familia, cumpliendo así una función de vertebrador social no calculado; éste es el caso de uno de los participantes quien comentó:

\footnotetext{
[...] pues de pronto la alegría, los pelados se han integrado más alrededor de la mascota, o sea, yo veo es la alegría y la unión familiar alrededor de la mascota, pues sí, los sentimientos muy unidos ahí frente a la mascota ( $\mathrm{S} 1$, comunicación personal, oct 20,2017).
}

Otros participantes manifestaron que las relaciones de los integrantes de la casa mejoraron debido a que tienen más temas de conversación o intereses en común; una participante afirmó: «[...] mi novio y yo no convivimos pero es como tener dos seres a los que los dos queremos y tenemos un nuevo tema de conversación siempre que hablamos, nos reímos juntos de cuenta de ellas» (S5, comunicación personal, feb 10, 2018), y otra sostuvo: «[...] a veces la relación de mi papá conmigo y con el gatico ha sido mucho mejor porque eso como que nos ha unido más y encontramos a veces muchas más cosas de que hablar juntos o pasar el tiempo juntos» (S8, comunicación personal, feb 5, 2018).

Adicionalmente, algunos participantes comentaron que ellos mismos o sus familiares se volvieron más tiernos, cariñosos y amorosos desde que empezaron a convivir con el animal que adoptaron. Un participante indicó: «[...] soy más tierno, más amoroso, más delicado, me siento más seguro» ( $\mathrm{S} 4$, comunicación personal, ene 5, 2018); otro expresó: «[...] a mi papá lo volvió más cariñoso» (S8, comunicación personal, feb 5, 2018). De igual forma, algunas personas percibieron que mejoraron sus relaciones sociales ya que se preocupan más por los animales en general y las demás personas que tienen a su alrededor; ante esto, una de las participantes declaró que «la responsabilidad del animalito... lo hace a uno pues como salir más a la calle, como socializar más con la gente... social, porque uno pues, no sé, se preocupa más como por las demás personas y los demás animalitos también» ( $\mathrm{S}$ 3, comunicación personal, nov 2, 2017).

En cuanto al impacto en el bienestar físico que ha tenido el animal de compañía en los adoptantes, se identificó que los participantes fueron físicamente 
más activos al velar por las necesidades de sus mascotas; una participante comentó al respecto:

[...] si hay que sacarlo a pasear, bueno, saquémoslo a pasear, que no se vuelva como una carga sino que es algo que la mascotica siente y que uno también disfrute al sacarlo, al servirle, al cambiarle el agua, servir el cuido, de que se acabó el cuido, ah, bueno, de que a uno no le dé pereza de ir al supermercado a conseguirlo, hacer las cosas como con gusto para tener bien al animalito (S9, comunicación personal, ene 5, 2018).

\section{Otra participante señaló que:}

[...] por él uno hace más ejercicio, entonces se siente uno como mejor de estado físico así no lo saquemos demasiado, pues que dos o tres horas en el día, pero sí, el hecho como tener uno de pronto la responsabilidad del animalito entonces lo hace a uno pues como salir más a la calle, como socializar más con la gente ( $\mathrm{S} 3$, comunicación personal, nov 2, 2017).

También se encontró que algunos participantes fueron más activos pues jugaban con sus animales de compañía; una participante sostuvo que: «[...] uno llega a la casa y lo primero que uno hace es pensar, ir [a] saludarlas, tiene entretenimiento, como jugar con ellas, estar con ellas» (S5, comunicación personal, feb 10, 2018), y otro participante expresó: «[...] pues a veces nos alegra mucho también porque se pone a jugar con nosotros» (S7, comunicación personal, feb 6, 2018).

Otros adoptantes manifestaron que sus mascotas han sido de gran ayuda para distraer cualquier tipo de dolor o enfermedad que puedan tener, por ejemplo, una participante indicó: «[...] me ha pasado que tengo un dolor de cabeza o algo y lo distraen a uno» (S2, comunicación personal, dic 12, 2017); igualmente, el participante $S 9$ expresó que la mascota tuvo un impacto en el bienestar físico de uno de sus familiares dado que:

[...] ahora la niña ha estado con dolorcito como en la pierna... pues ya ella se levanta, que tiene que sacar a pasear a la mascota, eh, precisamente hoy se levantó porque teníamos que ir a comprarle la camita a la mascota, pues que comprarle lo que el perrito necesitaba, entonces a ella la está empujando mucho a no pensar mucho en su dolor, sino que es algo que la tiene muy motivada y muy ocupada (S9, comunicación personal, ene 5, 2018). 
Sin embargo, se encontró los casos de dos participantes en los cuales la mascota tuvo un impacto físico negativo ya que uno expresó sentir desgaste y otro un deterioro en su salud; al respecto la participante S9 expresó: «[...] me ha desgastado un poquito, porque estos niños parecen locos con el perrito, todo el día lo quieren estar, todo el día quieren salir con el perro... entonces uno a veces como [que] repite, repite, a veces es desgastante» ( $S 9$, comunicación personal, ene 5, 2018). El participante $S 1$ indicó que: «[...] la señora dice que ella ha tenido quebrantos de salud por la mascota [...] ella le empezó una alergia y ha culpado a la mascota de la alergia» (S1, comunicación personal, oct 20, 2017).

Por otro lado, se halló que las mascotas tuvieron un impacto asociado a la disminución del estrés. Sobre este fenómeno algunos participantes expresaron sentirse más relajados debido a su animal de compañía. Una de las mujeres evaluadas manifestó lo que sigue:

[...] han cambiado cosas, como por ejemplo el manejo del estrés laboral que, pues, yo antes tenía el vicio de que llegaba a la casa de trabajar y era todo el tiempo, todo el tiempo pensando en el trabajo, en lo que había sucedido durante el día, en lo que tenía pendiente para el día siguiente y cosas así, y era como una costumbre que ya estaba cogiendo yo. Y desde que están las niñas todo es diferente porque ya uno llega a la casa y lo primero que uno hace es pensar, ir saludarlas, eh, tiene entretenimiento, como jugar con ellas, estar con ellas ( $\mathrm{S} 5$, comunicación personal, feb 10, 2018).

De igual manera, otro participante indicó que: «[En] un $100 \%$, me desestresa, me relaja» (S4, comunicación personal, ene 5, 2018). El impacto en el bienestar físico y emocional de la adopción de mascotas en los adoptantes se representa a continuación en la figura 2 . 
Figura 2. Impacto de la mascota tres meses después de su adopción

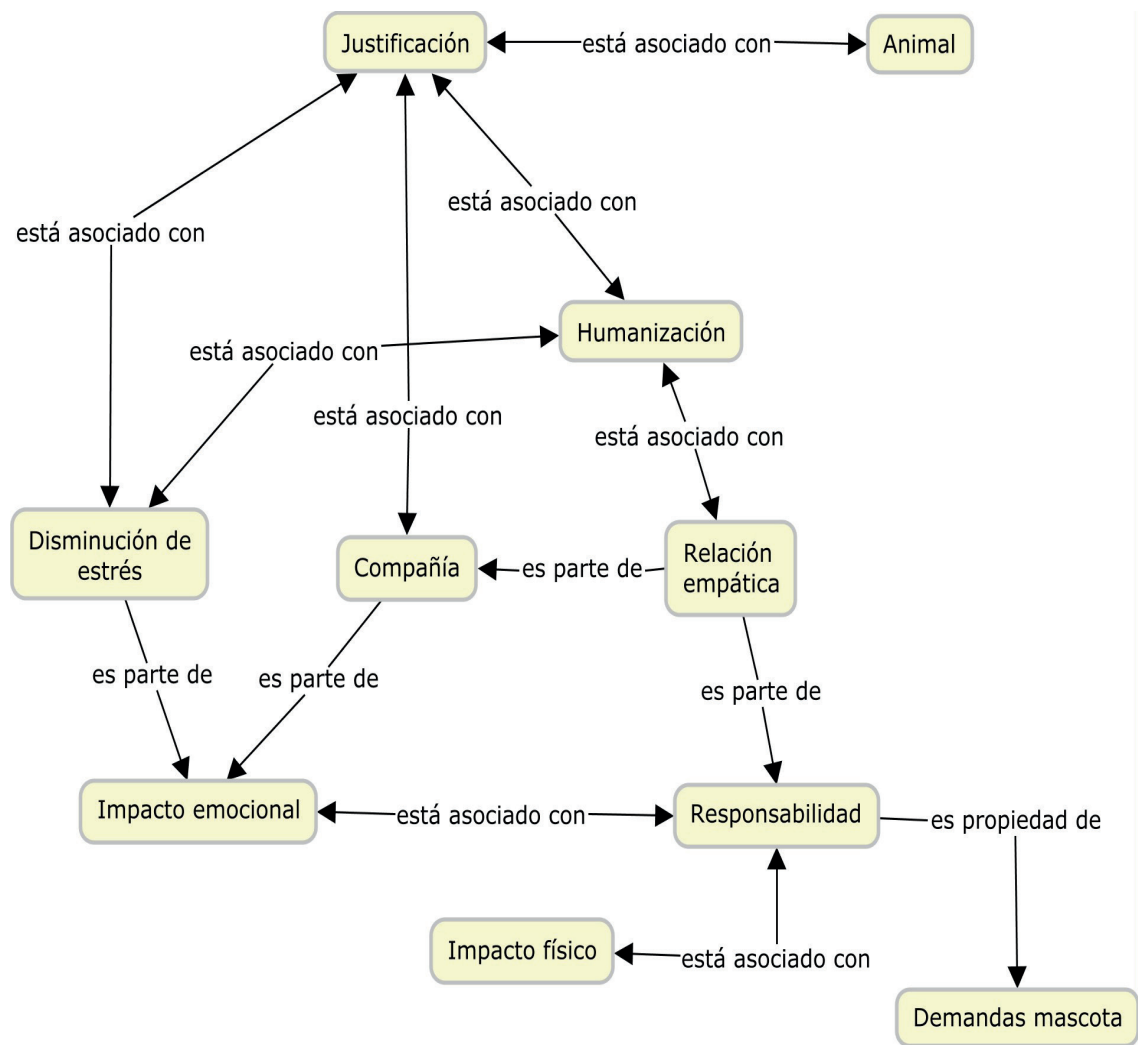

\section{Discusión}

Los resultados de este estudio evidencian cómo las mascotas pueden llegar a tener un impacto principalmente positivo en la percepción de bienestar por parte de los adoptantes, la cual parece estar relacionada con el rol asignado y la función que se les atribuye o se les da a los animales de compañía. Por un lado, se los puede ver como un animal que tiene por su presencia la capacidad de cumplir funciones pedagógicas o terapéuticas, por ejemplo, que lleve a com- 
prender el valor de la responsabilidad a partir de tener que asumirla mediante el cuidado de la mascota; función que podríamos denominarla como instrumental. Por otro lado, la mascota puede ser investida de valor en sí misma, la cual además ofrece compañía y opera como un vertebrador social al obligar a la interacción e integración social de las familias o grupos — de la tercera edad o infantiles, v. gr.- derivada de la necesidad de garantizar su cuidado; a esta función no calculada de la presencia de la mascota posiblemente podamos denominarla como trascendental.

Una de las principales razones de la adopción se centró en la compañía que los participantes en un primer momento consideraban que las mascotas les iban a brindar. Esto se asemeja a los motivos por los cuales las personas del país adoptan un animal de compañía, dado que, según un reportaje publicado en la Revista 4 Patas (2017), los colombianos tienden a adoptar un animal debido a la compañía incondicional que ofrecen, la sensación de cuidado y protección y por ser una poderosa fuente de distracción de las preocupaciones cotidianas.

Ahora bien, es importante resaltar que la compañía no es sólo el principal motivo para adoptar una mascota, sino también el principal beneficio derivado de su presencia; en este sentido la expectativa coincide con el resultado, lo cual probablemente contribuye aún más a percibir positivamente su impacto en la vida de los adoptantes, pues se sabe que se experimenta mayor satisfacción y menos frustración cuando lo esperado coincide con lo logrado.

Debe considerarse además que como consecuencia de los beneficios percibidos en la dimensión física y emocional, las mascotas tienden a ser humanizadas incluso por quienes en principio no las percibían así; desde luego, se profundiza esa percepción y tendencia en quienes de entrada ya las tenían humanizadas, como si de álter egos se tratara. Ello quizá se deba no sólo a una tendencia a la reciprocidad, sino al efecto de la tendencia social generalizada, acaecida en las últimas tres décadas, a desarrollar mayor nivel de sensibilidad hacia el medio ambiente, lo que incluye a los animales, quienes han dejado de ser vistos como seres inferiores o meros instrumentos para comenzar a ser reivindicados como sujetos de derecho. El movimiento ecologista, ambientalista y animalista parece haber tenido ya un efecto de sensibilización social frente al reconocimiento de la dignidad de los otros seres — flora y fauna-, supe- 
rándose así, por lo menos en parte, esa falsa visión de superioridad de los seres humanos respecto del resto de la naturaleza, actitud heredada de la tradición judeocristiana y reforzada por gran parte de la tradición científica.

Adicionalmente, se identificó que el rol que los adoptantes y sus familiares le otorgaron a las mascotas se relacionó directamente con los cambios que ellos percibieron en diferentes ámbitos de sus vidas a partir de la adopción de los animales de compañía. Al respecto se evidenció que la mayor parte de los participantes del presente estudio tuvieron la tendencia a humanizar a sus mascotas, incluso cuando su percepción inicial fue más "instrumentalista», por lo cual le atribuyeron a tales animales sentimientos, capacidades y actitudes propias de los humanos tales como la empatía, la ternura, capacidad de producir alegría, tranquilidad y confianza, así como de satisfacer la necesidad de reconocimiento.

En consecuencia, y en consonancia con esa tendencia espontánea a la humanización, se les consideró y trató como un miembro más de la familia. En estos casos, los participantes desarrollaron una relación empática y simpática con su mascota y a dicha relación se le atribuyó un impacto emocional generalmente positivo, ya que los participantes expresaron sentimientos de bienestar, confianza, alegría, tranquilidad e incluso se les adjudicó a las mascotas la capacidad de desestresar y generar seguridad; todo esto debido a que sentían que sus mascotas los acompañaban, les brindaban afecto, pero probablemente, sobre todo, porque les daban sentido a su vida, se volvían un asunto relevante del cual ocuparse y por el cual tenían que comunicarse, interactuar e integrarse con otros y hacer uso positivo de su tiempo libre.

El bienestar subjetivo está fundamentalmente asociado a la búsqueda de sentido y es posible que las mascotas al volverse un proyecto relevante para sí, que obliga en ocasiones a salir de sí mismo y a compartir con otros - función psicosocial que se comparte con el trabajo: la función de trascendencia, sentimiento de que se sale de sí para contribuir a algo más grande, a alguna perennidad, y de diversificación de la actividad, al ofrecer otros espacios para interactuar y hacer uso del tiempo y comprobar las capacidades- contribuye a esta percepción positiva de cómo se está en la vida y el mundo; en este sentido la mascota es un medio y un fin en sí misma. 
Por otro lado, se identificó que los adoptantes se perciben como físicamente más activos, sea por cumplir con las demandas de su mascota al tenerlos que sacar a caminar en el caso de quienes adoptaron perros, sea por jugar con sus mascotas; resultados que son similares a los resultados de otras investigaciones (Díaz y Olarte, 2016; Kushner et al., 2006; Lewis et al., 2009; Rodríguez, 2013; Lutz, 2013). Es importante anotar que estas investigaciones también habían encontrado que la tenencia de un animal de compañía podía llegar a incrementar la tendencia a sufrir alergias y asma. Si bien ninguno de los participantes del presente estudio notificó sufrir de asma, sí se evidenció el caso de una persona que consideró que desarrolló una alergia a raíz de la interacción con su mascota. Adicional a esto, en la presente investigación se encontró el caso de una participante que expresó haber sufrido un mayor desgaste físico al estar tan pendiente de su mascota junto con sus demás responsabilidades. Pero, en todo caso, se debe destacar que son más los efectos positivos que los negativos sobre la salud física y mental generados por las mascotas como consecuencia de su presencia en la vida de los adoptantes.

Respecto al impacto físico se encontró que la tenencia de un animal de compañía puede ser de gran ayuda para distraer algún tipo de dolor o constituir una motivación para superarlo Este resultado concuerda con otras investigaciones que probaron que la tenencia de un animal de compañía se relaciona con un incremento en la actividad física, consumo de comida más saludable, aumento en el cuidado de la salud y del optimismo de los dueños ante la enfermedad y la vida, fenómenos vinculados con una mejoría significativa del estado de salud percibido (López, 2015; Hugues et al., 2014; Núñez et al., 2014; Pacheco, 2012).

Asimismo, se halló que algunos participantes que interactuaban con sus mascotas identificaron una disminución significativa de los niveles de estrés que percibían antes de haber adoptado. Estos resultados coinciden con investigaciones realizadas sobre el tema. Por ejemplo, el impacto asociado a la disminución del estrés es semejante a la investigación realizada por Polherber y Matchock (2013), quienes identificaron que el interactuar con un animal de compañía —en este caso, un perro- disminuyó los niveles de cortisol en las personas expuestas a situaciones estresantes. 
La compañía en cuanto principal función y beneficio de las mascotas quizá tenga mucho sentido en el marco de una sociedad cada vez más regida por la ideología de la individualización (Beck y Beck-Gernsheim, 2003; Bauman, 2008) — variable simbólica_, en la que las personas están cada vez más empujadas a responder en solitario ante las contingencias de la existencia, pero en condiciones de mayor precariedad objetiva y subjetiva: la fragilización del lazo social es el ejemplo más contundente. Esta sensación de soledad que fuerza a diversas personas a adoptar una mascota para alejarse de sí o de un ser querido, no es sino el síntoma de una sociedad cada vez más individualista; súmese a esto la reducción significativa del tamaño real — tres miembros- y de configuración de los hogares - unipersonales en porcentajes crecientes en las ciudades más grandes (variable demográfica). Así pues, la protección ante las amenazas de la vida y la sensación de desamparo ya no es posible superarlas a través de la compañía de otros seres humanos semejantes, pues entre ellos todos tendemos a volvernos extraños y excesivamente condicionales; frente a una situación así parece ser que las mascotas, los animales que antes veíamos como inferiores, son un estrategia de defensa efectiva para afrontar esta nueva forma de experimentar el mundo.

Es lamentable que en el marco de una sociedad individualista, hiperproductivista y centrada en la acumulación sin límite, que nos pone a competir frenéticamente a todos contra todos, los seres humanos no podamos ya tejer relaciones de mutua confianza y reconocimiento recíproco entre nosotros (Honneth, 2003), que no tengamos ya confianza en la lealtad de los seres humanos, que en ese mundo los amigos tiendan a no tenerse más, que sean un bien simbólico en vía de extinción (Sennett, 2007; Enriquèz, 2000). Los animales, en condición de mascotas, tienen en sí mismos el potencial no sólo de servirnos de compañía, sino de obligarnos a interactuar e integrarnos con otros y desarrollar empatía y altruismo y experimentar mayor bienestar; por ese efecto parecen ser el antídoto al sinsentido de una sociedad cada vez más hiperindividualizada, solitaria.

En conclusión, se logró identificar que el rol que los participantes le otorgaron al animal de compañía influyó significativamente, a partir de la adopción, en los cambios percibidos en diversos aspectos de sus vidas, esto es, quienes 
humanizaron a sus mascotas desarrollaron una relación empática con éstas, la cual se vinculó significativamente con la compañía percibida y sentimientos de bienestar generados por sus mascotas, lo que generó un impacto emocional positivo para los adoptantes. De igual forma, se evidenció un impacto físico positivo debido a que los adoptantes se volvieron más activos físicamente al tener que suplir las demandas de sus mascotas. A su vez, se identificó que las mascotas se asociaron a la disminución del nivel de estrés presentado por algunos participantes; sin embargo, en dos casos el animal de compañía tuvo un efecto negativo en la salud.

Dado que el propósito de esta investigación se centró en identificar las razones de adopción y los beneficios recibidos en la salud física y emocional de los adoptantes de perros y gatos, consideramos conveniente indicar como limitaciones el hecho de que no se abordaron los motivos de adopción ni el impacto percibido en el bienestar físico y emocional de otros tipos de mascotas como las aves o algún otro tipo de animales domésticos. Tampoco se indagó por la percepción que se tiene de las mascotas y las razones de un determinado trato hacia ellas —en sentido recíproco- por parte de sus adoptantes, siendo esta variable ética y ecológica un asunto pendiente de investigar y no discutida aquí, pues superó el alcance del estudio. En tal sentido se recomienda desarrollar investigaciones similares con otros tipos de mascotas sobre el trato ofrecido a ellas en correspondencia con los sentidos construidos alrededor de lo que ellas son y significan para los seres humanos.

El valor práctico del estudio presente reside en mostrar las motivaciones y la función psicosocial que pueden cumplir las mascotas para los seres humanos, y cómo ellas pueden ser útiles para el desarrollo de la empatía, las actitudes altruistas, el establecimiento de relaciones de reconocimiento recíproco, así como para la facilitación de la integración social a partir de la constitución del cuidado de la mascota como un proyecto común. Todo esto puede servir para desarrollar estrategias pedagógicas, terapéuticas y de intervención psicosocial con familias y grupos - de niños o adultos de la tercera edad- en contextos institucionalizados y no institucionalizados, o en contextos clínicos y no clínicos. Si bien hay suficiente evidencia del impacto positivo de los animales en el campo de la terapia, este estudio por sus resultados nos invita a 
considerar a las mascotas como recurso de intervención en contextos no sólo terapéuticos sino cotidianos.

\section{REFERENCIAS}

Ávila, A., Torres, G., Villazala, O., López, A., Moldes, I., Saavedra, M., Larrañeta, L., y Pérez, L. (2013). Efectos de la terapia asistida con animales en personas con daño cerebral o lesión medular. Trauma, 24(2), 109-116.

Bauman, Z. (2008). A sociedade individualizada: vidas contadas e histórias vividas. Rio de Janeiro: Zahar.

Beck, U., y Beck-Gernsheim, E. (2003). La individualización: el individualismo institucionalizado y sus consecuencias sociales y políticas. Barcelona: Paidós.

Cabra, C., Hincapié, S., Jiménez, D., y Tobón, M. (2011). Estudio descriptivo de los efectos que ejerce el perro como mascota en el desarrollo de la motricidad gruesa de infantes sanos de cinco años de edad. Revista Lasallista de Investigación, 8(1), 82-89.

Díaz, M., y Olarte, M. (2016). Animales de compañía, personalidad humana y los beneficios percibidos por los custodios. Revista Latinoamericana de Ciencia Psicológica, 8(2), 1-21.

Durán, M. (2010). Bienestar psicológico: el estrés y la calidad de vida en el contexto laboral. Revista Nacional de Administración, 1(1), 71- 84.

Enriquèz, E. (2000). «O indivíduo preso na armadilha da estrutura estratégica». En: F. Mottas y M. De Freitas (eds.). Vida psíquica e organização. São Paulo: Editora FGV.

García, C., Zamorano, E., Ruiz, M., Pardo, A., Pérez, P., López, V., Freire, O., y Rejas, J. (2010). Cultural adaptation into Spanish of the generalized anxiety disorder-7 (GAD-7) scale as a screening tool. Health Qual Life Outcomes, 8(8), I-I I.

Gonzáles, M., y Landero, R. (2011). Diferencias en estrés percibido, salud mental y física de acuerdo al tipo de relación humano-perro. Revista Colombiana de Psicología, 20(1), 75-86.

Fonseca, H. (2010). Efectos de la zooterapia canina en las conductas prosociales y el nivel de actividad fisica de un niño con trastorno autista y sobrepeso (Tesis de maestría). Campus presbítero Benjamín Heredia, Costa Rica. 
Honneth, A. (2003). Luta por reconhecimento: a gramática moral dos conflitos sociais. São Paulo: Editora 34.

Hugues, B., Álvarez, A., Castelo, L., Ledón, L., Mendoza, M., y Domínguez, E. (2014a). Percepción de los beneficios de la tenencia de animales de compañía en tres grupos poblacionales de La Habana, Cuba. Revista de Investigaciones Veterinarias del Perú, 25(3), 355-365.

Hugues, B., Álvarez, A., Castelo, L., Ledón, L., Mendoza, M., y Domínguez, E. (2014b). Percepción de los beneficios de la tenencia de animales de compañía en pacientes con enfermedades cardiovasculares. CorSalud, 6(1), 56-62.

Hugues, B., Álvarez, A., Castelo, L., Ledón, L., Mendoza, M., y Domínguez, E. (2016). Percepción de los beneficios de los animales de compañía para adultos mayores con diabetes mellitus tipo 2. Revista de Investigaciones Veterinarias del Perú, 27(2), 233-240.

Ivancevich, J., y Matteson, M. (1992). Estrés y trabajo: una perspectiva gerencial. México: Editorial Trillas.

Kushner, R., Jackson, D., Jewell, D., y Rudloff, K. (2006). The PpeT study: people and pets exercising together. Obesity, 14(10), 1762-1770.

Lewis, A., Krägeloh, C., y Shepherd, D. (2009). Pet Ownership, Attachment and Health-Rated Quality of Life in New Zealand. Electronic Journal of Applied Psychology, 5(1), 96-101.

López, F. (2015). Influencia de las mascotas en la calidad de vida de los adultos mayores (Tesis de pregrado). Universitas Miguel Hernández, España.

López, M., Filippetti, V., y Richaud, M. (2013). Empatía: desde la percepción automática hasta los procesos controlados. Avances en Psicología Latinoamericana, 32(1), 37-51.

Lutz, R. (2013). Walking the dog: the effect of pet ownership on human health and health behaviors. Springer Science+business Media, 116(2), 327-339.

Núñez, F., León, M., Morales, M., y Roa, M. (2004). La relación del adulto mayor con los animales de compañía en una comuna de Santiago. Avances en Ciencias Veterinarias, 19(2), 75-79.

Orejuela, J. (2018). Clínica del trabajo: el malestar subjetivo derivado de la fragmentación laboral. Bogotá: San Pablo-EAfiT.

Organización Mundial de la Salud [oms] (2015). ¿Cómo define la OMS la salud? Organización Mundial de la Salud. Recuperado de http://www.who.int/suggestions/ faq/es/ 
Pacheco, A. (2012). Therapeutic Profit by Companion Animals Employment in Health Care of Older People. Revista Gerencia y Politicas de Salud, 11(22), 58-66.

Pineda, E. (2008). La delfinoterapia en niños con síndrome Down atendidos en un círculo infantil especial. Alegría, 38, 1-7.

Polheber, J., y Matchock, R. (2013). The presence of a dog attenuates cortisol and heart rate in the trier social stress test compared to huma friends. Springer Science+business Media, 37(5), 860-867.

Ramos, A., Díaz, Y., Báez, F., y Tarajano, A. (2006). Equinoterapia: experiencia en un ańo de tratamiento. Revista Archivo Médico de Camagüey, 10(6), 102-111.

Redacción Economía El Espectador (28 de enero de 2015). En seis de cada diez hogares colombianos hay mascotas. El Espectador. Recuperado de https://www.elespectador.com/noticias/economia/seis-de-cada-10-hogares-colombianos-hay-mascotas-articulo-540449

Revista 4 Patas. Las mascotas, la mejor cura para la depresión. (2017). Revista 4 Patas. Recuperado de http://www.4patas.com.co/perros/comportamiento/articulo/ los-perros-ayudan-a-las-personas-tristes/3814

Rodríguez, M. (2013). Los perros como catalizadores de capital social, salud y actividad: Estudio en la comunidad cercana al parque para perros de Frases de Curridabat (Tesis de pregrado). Ciudad Universitaria Rodrigo Facio, Costa Rica.

Rodríguez, M., y Muñoz, R. (2010). Influencia de tener perros sobre la salud percibida en personas mayores de Jaén (España). Revista Colombiana de Enfermería, 11, 29-33.

Sennett, R. (2007). La cultura del nuevo capitalismo. Barcelona: Anagrama.

Urzúa, M. (2012). Calidad de vida: una revisión teórica del concepto. Terapia Psicológica, 30(1), 61-71. 